\title{
Market Microstructure and Price Discovery
}

\author{
Paul Carlisle Kettler ${ }^{1}$, Aleh L. Yablonski ${ }^{1,2}$, Frank Proske ${ }^{1}$ \\ ${ }^{1}$ Centre of Mathematics for Applications, Department of Mathematics, University of Oslo, Oslo, Norway \\ ${ }^{2}$ Department of Functional Analysis, Mechanical and Mathematical Faculty, \\ Belarusian State University, Minsk, Belarus \\ Email: paulck@math.uio.no,yablonski@bsu.by, proske@math.uio.no
}

Received July 13, 2012; revised September 18, 2012; accepted October 4, 2012

\begin{abstract}
The design of this study is to investigate the evolution of a stochastic price process consequent to discrete processes of bids and offers in a market microstructure setting. Under a set of flexible assumptions about agent preferences, we generate a price process to compare with observation. Specifically, we allow for both rational and irrational economic behavior, abstracting the inquiry from classical studies relying on utility theory. The goal is to provide a set of economic primitives which point inexorably to the price processes we see, rather than to assume such process from the start.
\end{abstract}

Keywords: Price Theory and Market Microstructure; Stochastic Difference Equations; Bid; Ask; Price Processes in Discrete Time

\section{Introduction}

We propose to model a price process based on microstructural activity of a market. We assume a set of agents such that each agent at any moment has both bid and ask prices present in the market. A trade occurs if and only if the bid of one agent is equal to the ask of another, this common value becoming the price of a trade. We calculate the dynamics of the resulting price process, including the moments of trades, in a discrete time setting for behavioral choices of the agents. These choices are formalized in relevant probability distributions specific to the agents' behaviors. In this way, we allow for a multitude of behavioral patterns, including, but not restricted to traditional motivations inspired by utility functions. Our model is flexible enough to allow for "marks" to a trade, ancillary data such as its time stamp, so that we may study independently such features as trade clustering and time deformation.

Recent history is rich with microstructure studies of financial markets and with associations of specific families of probability distributions to financial stochastic processes. For good reviews of the microstructure literature see these works respectively [1,2]. For associations of probability distributions such as the widely applied Gaussian, normal inverse Gaussian, and more inclusively the generalized hyperbolic, see these studies [3,4]. In many instances such inquiries assume at the outset various forms of stochastic processes, as defined by stochastic differential equations, and then set forth to esti-

*The work of Aleh L. Yablonski was supported by INTAS grant 03-551861. mate parameters. Popular choices are Itô diffusions and Ornstein-Uhlenbeck processes, with and without the superposition of pure jump Lévy processes.

Most studies of microstructure take an econometric approach, that is, they define some structure, assume distributions as appropriate, then estimate parameters using data. In his survey with important bibliography, Bollerslev reviews the state of financial econometrics [5]. In a subsection discussing time-varying volatility, he notes that, "several challenging questions related to the proper modeling of ultra high-frequency data, longer-run dependencies, and large dimensional systems remain.” Further in the text, he qualifies this remark by stating: "Not withstanding much recent progress, the formulation of a workable dynamic time series model which readily accommodates all of the high-frequency data features, yet survives under temporal aggregation, remains elusive.”

Engle provides just such an econometric study [6] employing the Autoregressive Conditional Duration (ACD) model developed by him with Russell [7] in the study of IBM stock transactional arrival times. In the former paper, Engle, in referring to cases of the conditional duration function, relates, "In each case, the density is assumed to be exponential." Such assumptions are typical, and necessary, for an econometric study focusing on time series of prices as the fundamental data structure.

Hasbrouck, in focusing on the refinement of bid and ask quotes, proposes and estimates an Autoregressive Conditional Heteroskedasticity (ARCH) model using 
Alcoa stock transactions, evenly spaced at 15 minute intervals [8]. Routinely, he asks the reader to consider, "a stock with an annual log return standard deviation of 0.30 " The reference "return" is of course to the price sequence, a necessary expedient in the classical econometric framework which considers a price process as fundamental, rather than consequential to a set of underlying bid and ask processes.

Other studies, such as one by Bondarenko, delve into the bid and ask series, but rather as a difference, the spread [9]. The focus of this work and its principal results are in the realm of market liquidity, rather than in the estimation of the price process. Once again, the classical framework requires an assumption on the distribution of the price process, as evidenced in this remark made within the context of evaluating a price change between periods. "The asset's final value is denoted $v^{*}$, a normal random variable with mean $v_{0}$ and variance $\sigma_{v}^{2}$."

Yet further studies attempt to develop directly a price process from first principles. An interesting and provocative example is a paper by Schaden, which formulates conclusions from financial analogues to fundamentals of quantum physics [10]. As he observes in the introduction, "At this stage it is impossible to decide whether a quantum description of finance is fundamentally more appropriate than a stochastic one, but quantum theory may well provide a simpler and more effective means of capturing some of the observed correlations." Indeed, though the basic process investigated is yet a price process, not those of bids and asks. The analysis is grounded on five at first qualitative assumptions about the market, and concludes with the assertion that the evolution of prices follows "the lognormal price distribution." In this setting it is difficult to discern how a different-and more realistic - distribution could emerge without changing substantially the assumptions, or the physics. For further background reading see [11-13].

In our paper we choose to move to a more basic level of explanation, to specify the market mechanisms among interacting agents, and then to let the model determine the price process and its features. In this way we derive such features as the distributions of prices, rather than assuming them $a b$ initio.

We now proceed forthwith to present our case.

\section{Specification of the Model}

We consider for simplicity the model of the market for one stock in discrete time $t \in \mathbf{T}=\{0,1, \cdots, T\}^{1}$. It is reasonable to assume that in each time $t \in \mathbf{T}$ there are only finite number $n_{t}$ of agents taking part in the trad-

${ }^{1}$ For a treatment of the case wherein the duration, defined as the length of time between trades, is stochastic, see [14]. ing on the market. Let $N$ be the number of all agents which have ever taken part in trading. At each moment $t \in \mathbf{T}$ the agent number $i, \quad 1 \leq i \leq N$ proposes a bid price $b_{t}^{i}$ and an ask price $a_{t}^{i}$ for a goods on the market. We assume that $a_{t}^{i} \geq b_{t}^{i}$. It is convenient to set $a_{t}^{i}=\infty$ and $b_{t}^{i}=0$ if at the moment $t \in \mathbf{T}$ the $i$-th agent does not take part in the trading. Supposing the rational behavior of agents on the market we have $A_{t} \geq B_{t}$, where $A_{t}=\min \left\{a_{t}^{i}: 1 \leq i \leq N\right\}$ and $B_{t}=\max \left\{b_{t}^{i}: 1 \leq i \leq N\right\}$. We say that there is a trade between $i$-th and $j$-th agents at moment $t \in \mathbf{T}$ if $a_{t}^{i}=A_{t}=B_{t}=b_{t}^{j}$ or $a_{t}^{j}=A_{t}=B_{t}=b_{t}^{i}$. It means that there is a trade between agents with minimal ask price $A_{t}$ and maximal bid price $B_{t}$ provided that they are equal $A_{t}=B_{t}$. In order to escape some pathological examples we always assume that at every time $t$ there exist two different agents, say number $i$ and $j, i \neq j$, such that $a_{t}^{i}=A_{t}$ and $b_{t}^{j}=B_{t}$. In the case when more than one of the agents have the same minimal ask price and maximal bid price, say $A_{t}=a_{t}^{i_{1}}=\cdots=a_{t}^{i_{n}} \quad$ and $B_{t}=b_{t}^{j_{1}}=\cdots=b_{t}^{j_{n}}$, we suppose that a trade occurs between agents with numbers $i_{1}, \cdots, i_{k}$ and $j_{1}, \cdots, j_{k}$, where $k=m \wedge n$.

The bids and asks can be changed only by the agents. It may happen that $A_{t}<B_{t}$ after such changing of prices. In order to avoid such possibilities we suppose that bid prices can be changed by agents only at even moments and ask prices only at odd moments. Nevertheless the trades can occur at any moment: even or odd.

How should the bid and ask prices change? The rules of changing bid and ask prices by the agents are different for each agent and they are based on different reasons; for instance: aims of agents, interpretations of information, personal reasons, and so on. If these prices are changed at time $t$ when a trade occurs, say between the $i$-th and $j$-th agents with prices $a_{t}^{i}=b_{t}^{j}=A_{t}=B_{t}$, then the respective ask price $a_{t+1}^{i}$ will be not less then the price before the trade $a_{t}^{i} \leq a_{t+1}^{i}$. Therefore we can say that

$$
a_{t+1}^{i}=a_{t}^{i} \mathrm{e}^{\alpha_{t}^{i}}=B_{t} \mathrm{e}^{\alpha_{t}^{i}},
$$

where $\alpha_{t}^{i}$ is a nonnegative random variable (it is possible to add one more value $\infty$ if the agent decides to leave the market). For the bid prices we can write similarly

$$
b_{t+1}^{j}=b_{t}^{j} \mathrm{e}^{-\beta_{t}^{j}}=A_{t} \mathrm{e}^{-\beta_{t}^{j}},
$$

with nonnegative random variable $\beta_{t}^{j}$ (with the same note about $\infty$ ). The random variables $\alpha_{t}^{i}$ and $\beta_{t}^{j}$ are $\mathcal{F}_{t}^{i}$ - and $\mathcal{F}_{t}^{j}$-adapted, respectively, where $\mathcal{F}_{t}^{i}$ and $\mathcal{F}_{t}^{j}$ are $\sigma$-fields containing information which the agents know before the time $t$, inclusively. Note that $\alpha_{t}$ and $\beta_{t}$ are defined only at the moment $t$ of trades.

As in the previous case we can write the same equ- 
alities for a moment $t$ when the respective agent was not involved in a trade. Hence for any $t \in \mathbf{T}$ we have

$$
a_{t+1}^{i}=B_{t} \mathrm{e}^{\alpha_{t}^{i}} \text { and } b_{t+1}^{i}=A_{t} \mathrm{e}^{-\beta_{t}^{i}},
$$

where $\alpha_{t}^{i}$ and $\beta_{t}^{i}, \quad i=1,2, \cdots, N$ are nonnegative random variables. The moment $\tau_{t}$ and the price $S_{t}$ of the last trade before time $t=1,2, \cdots$ inclusively are given by

$$
\tau_{t}=\sup \left\{0<s \leq t: A_{s}=B_{s}\right\} \text { and } S_{t}=A_{\tau_{t}}\left(=B_{\tau_{t}}\right) .
$$

Set $\tau_{0}=0$ and $S_{0}=1$.

The purpose of present paper is to calculate the distributions of $\tau_{t}$ and $S_{t}$ from Equation (2.2) by using the known distributions of $a_{t}$ and $b_{t}$ from Equations (2.1).

Taking min and max in Equations (2.1) yields

$$
A_{t+1}=B_{t} \mathrm{e}^{\mu_{t}} \text { and } B_{t+1}=A_{t} \mathrm{e}^{-v_{t}} \text {, }
$$

where $\mu_{t}=\min \left\{\alpha_{t}^{i}: 1 \leq i \leq N\right\}$ and $v_{t}=\min \left\{\beta_{t}^{i}: 1 \leq i \leq N\right\}$ are nonnegative random variables. Notice that $\mu_{t}$ and $v_{t}$ are $\mathcal{F}_{t}$-measurable, where $\mathcal{F}_{t}=\sigma\left\{\mathcal{F}_{t}^{i}: 1 \leq i \leq N\right\}$ is information known to at least one agent before time $t$, inclusively.

Let us consider two nonnegative random processes $X_{t}=A_{t} B_{t}$ and $Y_{t}=A_{t} / B_{t}$. From Equalities (2.3) we deduce that

$$
\begin{gathered}
X_{t+1}=X_{t} \mathrm{e}^{\mu_{t}-v_{t}}, \\
Y_{t+1}=\mathrm{e}^{\mu_{t}+v_{t}} / Y_{t} .
\end{gathered}
$$

Since the trade occurs at the moment $t$ if and only if $A_{t}=B_{t}$ or, equivalently, if $Y_{t}=1$, then the last moment of a trade before the time $t$

$$
\tau_{t}=\sup \left\{0<s \leq t: Y_{s}=1\right\}
$$

is the last moment before $t$ when the process $Y_{t}$ reached the level 1 . The price of the last trade before the time $t$ is given by

$$
S_{t}=\sqrt{X_{\tau_{t}}} .
$$

Now the problem is reduced to finding the law of random time $\tau_{t}$ given by (2.6) and the law of the process $X_{t}$ given by Equation (2.4) at the time $\tau_{t}$.

\section{Simplest Behavior of Agents}

Since the bid prices can be changed by the agent in even moments only, then $B_{2 k+1}=B_{2 k}$. Therefore from Equation (2.3) we deduce that

$$
v_{2 k}=\log \left(A_{2 k} / B_{2 k}\right) .
$$

Similarly $A_{2 m}=A_{2 m-1}$ and

$$
\mu_{2 m-1}=\log \left(A_{2 m-1} / B_{2 m-1}\right) .
$$

Then Equations (3.1), (3.2) and (2.5) imply that $Y_{2 k}=\mathrm{e}^{\nu_{2 k-1}}$ and $Y_{2 k+1}=\mathrm{e}^{\mu_{2 k}}$. Moreover, we have $v_{2 k-1}=v_{2 k}$ and $\mu_{2 k}=\mu_{2 k+1}$. Define a new sequence $\xi_{t}$ by $\xi_{t}=v_{t-1}$ for $t=2 k$ and $\xi_{t}=\mu_{t-1}$ if $t=2 k-1$, $k=1,2, \cdots$. Then $\xi_{t} \geq 0, Y_{t}=\mathrm{e}^{\xi_{t}}$ and $\tau_{t}=\sup \left\{0<s \leq t: Y_{s}=1\right\}=\sup \left\{0<s \leq t: \xi_{t}=0\right\}$. Hence the trade occurs at time $t$ if and only if $\xi_{t}=0$.

In order to obtain some result we need to have more assumptions on the behavior of the processes $\mu$ and $v$. The simplest assumption is that $\xi_{t}, t=1,2, \cdots$ is a sequence of independent identically distributed (i.i.d.) random variables. Denote by $p$ the probability that $\xi_{1}$ takes value zero: $p=\operatorname{Pr}\left[\xi_{1}=0\right]$. The variable $\tau_{t}$ is a last zero of the sequence $\xi$ before the moment $t$. We put $\tau_{t}=0$ if there are no zeros (no trades) before time $t$, inclusively. Hence $\tau_{t}$ takes values $0, \cdots, t$. The probabilities of these values are given by

$$
\begin{aligned}
\operatorname{Pr}\left[\tau_{t}=0\right] & =\operatorname{Pr}\left[\xi_{1}>0 ; \xi_{2}>0 ; \cdots ; \xi_{t}>0\right] \\
& =\left[\operatorname{Pr}\left[\xi_{1}>0\right]\right]^{t}=(1-p)^{t},
\end{aligned}
$$

and for $k=1, \cdots, t$

$$
\begin{aligned}
\operatorname{Pr}\left[\tau_{t}=k\right] & =\operatorname{Pr}\left[\xi_{k}=0 ; \xi_{k+1}>0 ; \cdots ; \xi_{t}>0\right] \\
& =p(1-p)^{t-k} .
\end{aligned}
$$

Let $M_{t}, t=1,2, \cdots$ denote the number of trades before time $t$ inclusively. Hence $M_{t}$ is number of zeros in the sequence $\xi_{k}, k=1,2, \cdots, t$. Then $M_{t}$ has a binomial distribution with parameters $p$ and $t$, i.e.,

$$
\operatorname{Pr}\left[M_{t}=k\right]=\left(\begin{array}{l}
t \\
k
\end{array}\right) p^{k}(1-p)^{t-k}, k=0,1, \cdots, t
$$

here $\left(\begin{array}{l}t \\ k\end{array}\right)=t ! /(k !(t-k) !)$ is a binomial coefficient.

Moreover $M_{t+s}-M_{s}$ has a binomial distribution with the same parameters $p$ and $t$. As a consequence of independence of the variables $\xi_{t}$ we get that for any $0 \leq t_{0} \leq t_{1} \leq \cdots \leq t_{m} \leq T$ the random variables $M_{t_{0}}$, $M_{t_{1}}-M_{t_{0}}, \cdots, M_{t_{m}}-M_{t_{m-1}}$ are independent.

Define the sequence $\sigma_{k}, t \in \mathbf{T}$ of random times inductively by the following expression.

$$
\sigma_{k}=\inf \left\{t>\sigma_{k-1}: \xi_{t}=0\right\},
$$

with $k=1,2, \cdots$ and $\sigma_{0}=0$. We adopt the convention that the infinum of empty set is equal to infinity. Then $\sigma_{k}, k=1,2, \cdots$ is a moment of $k$-th trade (or zero of the sequence $\xi_{t}$ ) and

$$
\begin{aligned}
\left\{\sigma_{k}=m\right\} & =\left\{M_{m}=k ; M_{m-1}=k-1\right\} \\
& =\left\{M_{m}-M_{m-1}=1 ; M_{m-1}=k-1\right\}
\end{aligned}
$$

for $m=k, k+1, k+2, \cdots, T$. Easy calculation shows that 


$$
\begin{aligned}
& \operatorname{Pr}\left[\sigma_{k}=m\right]=\operatorname{Pr}\left[M_{m}-M_{m-1}=1 ; M_{m-1}=k-1\right] \\
& =\left(\begin{array}{c}
m-1 \\
k-1
\end{array}\right) p^{k}(1-p)^{m-k}, m=k, k+1, \cdots, T
\end{aligned}
$$

and

$$
\begin{aligned}
\operatorname{Pr}\left[\sigma_{k}=\infty\right] & =\operatorname{Pr}\left[M_{T} \leq k-1\right] \\
& =\sum_{j=0}^{k-1}\left(\begin{array}{c}
T \\
j
\end{array}\right) p^{j}(1-p)^{T-j} .
\end{aligned}
$$

Furthermore for all $1 \leq m_{1}<m_{2}<\cdots<m_{k} \leq T$, $k=1,2, \cdots, T$ we have

$$
\begin{aligned}
& \operatorname{Pr}\left[\sigma_{1}=m_{1} ; \sigma_{2}=m_{2} ; \cdots ; \sigma_{k}=m_{k}\right] \\
& =p^{k}(1-p)^{m_{k}-k}
\end{aligned}
$$

and

$$
\begin{aligned}
& \operatorname{Pr}\left[\sigma_{1}=m_{1} ; \sigma_{2}=m_{2} ; \cdots ; \sigma_{k}=m_{k} ; \sigma_{k+1}=\infty\right] \\
& =p^{k}(1-p)^{T-k} .
\end{aligned}
$$

For any $k=1,2, \cdots$ and $m=1,2, T-k$ we have

$$
\operatorname{Pr}\left[\sigma_{k+1}-\sigma_{k}=m\right]
$$$$
=\sum_{j=k}^{T-m} \operatorname{Pr}\left[\sigma_{k}=j ; \sigma_{k+1}=m+j\right]
$$$$
=\sum_{j=k}^{T-m} \sum_{\substack{1 \leq m_{1}<m_{2}<j<m_{k-1}<j \\<-1}} \operatorname{Pr}\left[\sigma_{1}=m_{1} ; \sigma_{2}=m_{2} ; \cdots ; \sigma_{k}=j ; \sigma_{k+1}=m+j\right]
$$$$
=\sum_{j=k}^{T-m}\left(\begin{array}{c}
j-1 \\
k-1
\end{array}\right) p^{k+1}(1-p)^{j+m-k-1}
$$$$
=p^{k+1}(1-p)^{m-1} \sum_{j=0}^{T-m-k}\left(\begin{array}{c}
j+k-1 \\
k-1
\end{array}\right)(1-p)^{j}
$$

and

$$
\begin{aligned}
& \operatorname{Pr}\left[\sigma_{k+1}-\sigma_{k}=\infty\right]=\operatorname{Pr}\left[\sigma_{k+1}=\infty\right] \\
& =\operatorname{Pr}\left[M_{T} \leq k\right]=\sum_{j=0}^{k}\left(\begin{array}{c}
T \\
j
\end{array}\right) p^{j}(1-p)^{T-j} .
\end{aligned}
$$

In the same way one can obtain

$$
\begin{aligned}
& \operatorname{Pr}\left[\sigma_{k+1}-\sigma_{k}=n_{1} ; \sigma_{k}-\sigma_{k-1}=n_{2}\right] \\
& =\sum_{j=k-1}^{T-n_{1}-n_{2}} \sum_{1 \leq m_{1}<m_{2}<\cdots<m_{k-2}<j} p^{k+1}(1-p)^{j+n_{1}+n_{2}-k-1} \\
& =\sum_{j=k-1}^{T-n_{1}-n_{2}}\left(\begin{array}{c}
j-1 \\
k-2
\end{array}\right) p^{k+1}(1-p)^{j+n_{1}+n_{2}-k-1} \\
& =p^{k+1} \sum_{j=0}^{T-n_{1}-n_{2}-k+1}\left(\begin{array}{c}
k+j-2 \\
k-2
\end{array}\right)(1-p)^{j+n_{1}+n_{2}-2} .
\end{aligned}
$$

Notice that

$$
\begin{aligned}
& \operatorname{Pr}\left[\sigma_{k+1}-\sigma_{k}=n_{1} ; \sigma_{k}-\sigma_{k-1}=n_{2}\right] \\
& \neq \operatorname{Pr}\left[\sigma_{k+1}-\sigma_{k}=n_{1}\right] \operatorname{Pr}\left[\sigma_{k}-\sigma_{k-1}=n_{2}\right]
\end{aligned}
$$

Hence $\sigma_{k+1}-\sigma_{k}$ and $\sigma_{k}-\sigma_{k-1}$ are not independent.

Let us consider process $X_{t}$ given by Equation (2.4). The solution of this equation can be written as

$$
X_{t}=X_{0} \exp \left(\sum_{k=0}^{t-1}\left(\mu_{k}-v_{k}\right)\right) .
$$

Since $v_{2 k-1}=v_{2 k}=\xi_{2 k}$ and $\mu_{2 k}=\mu_{2 k+1}=\xi_{2 k+1}$ then

$$
\begin{aligned}
\sum_{k=0}^{t-1}\left(\mu_{k}-v_{k}\right) & =\sum_{k=0}^{t-1}\left(\xi_{2\left[\frac{k}{2}\right]+1}-\xi_{2\left[\frac{k+1}{2}\right]}\right) \\
& =2 \sum_{k=1}^{t}(-1)^{k+1} \xi_{k}+(-1)^{t} \xi_{t}-v_{0},
\end{aligned}
$$

where $[m]$ denotes the integer part of number $m$.

Therefore taking into account that $v_{0}=\log \left(Y_{0}\right)$ one has

$$
X_{t}=\frac{X_{0}}{Y_{0}} \exp \left((-1)^{t} \xi_{t}+2 \sum_{k=1}^{t}(-1)^{k+1} \xi_{k}\right) .
$$

From the Equation (3.4) and definition of $X_{0}$ and $Y_{0}$ we obtain the prices $S_{t}$ and $S^{(k)}$ of the last trade and the $k$-th trade:

$$
\begin{aligned}
& S_{t}=\sqrt{X_{\tau_{t}}} \\
& \quad=B_{0} \exp \left(\sum_{j=1}^{\tau_{t}}(-1)^{j+1} \xi_{j}+(-1)^{\tau_{t}} \xi_{\tau_{t}} / 2\right) . \\
& S^{(k)}=\sqrt{X_{\sigma_{k}}} \\
& =B_{0} \exp \left(\sum_{j=1}^{\sigma_{k}}(-1)^{j+1} \xi_{j}+(-1)^{\sigma_{k}} \xi_{\sigma_{k}} / 2\right) .
\end{aligned}
$$

Now we calculate the characteristic function $f_{t}(z)$ of the logarithm $\log \left(S_{t} / B_{0}\right)$. It follows from representation (3.5) that

$$
\begin{aligned}
& f_{t}(z)=E\left[\exp \left(\mathrm{i} z \log \left(S_{t} / B_{0}\right)\right)\right] \\
& =\sum_{k=0}^{t} E\left[\exp \left(\mathrm{i} z \log \left(\sqrt{X_{\sigma_{k}}} / B_{0}\right)\right) 1_{\left\{\sigma_{k} \leq t<\sigma_{k+1}\right\}}\right] \\
& =\operatorname{Pr}\left[t<\sigma_{1}\right] \\
& +\sum_{k=1}^{t} \sum_{\substack{1 \leq m_{1}<m_{2}<\cdots \\
<m_{k} \leq t<m_{k+1}}} E\left[\exp \left(\mathrm{i} z \log \left(\sqrt{X_{\sigma_{k}}} / B_{0}\right)\right) 1 \prod_{j=1}^{k+1}\right]
\end{aligned}
$$

Notice that event $\left\{\sigma_{1}=m_{1} ; \cdots ; \sigma_{k}=m_{k}\right\}$ occur if and only if $\xi_{m_{1}}=\xi_{m_{2}}=\cdots=\xi_{m_{k}}=0$ and $\xi_{j}>0$ if $j$ does not coincide with some of the $\left\{m_{i}\right\}$. This fact, formula (3.5), independence and the distribution of $\xi_{i}$ imply 


$$
\begin{aligned}
f_{t}(z) & =\operatorname{Pr}\left[t<\sigma_{1}\right]+\sum_{k=11 \leq m_{1}<m_{2}<\cdots<m_{k} \leq t<m_{k+1}}^{t} E\left[\prod_{j=1}^{k+1} 1_{\left\{\xi_{m_{j}}=0\right\}} \prod_{j=1, j \neq m_{1}, m_{2}, \cdots, m_{k}} y^{m_{k+1}-1} 1_{\left\{\xi_{j}>0\right\}} \times \exp \left(i z \sum_{j=1}^{m_{k}}(-1)^{j+1} \xi_{j}+(-1)^{m_{k}} \xi_{m_{k}} / 2\right)\right] \\
& \left.=(1-p)^{t}+\sum_{k=11 \leq m_{1}<m_{2}<\cdots<m_{k} \leq t}^{t} \sum_{m_{k+1}=t+1} p^{k+1}(1-p)^{m_{k+1}-m_{k}-1}+p^{k}(1-p)^{T-m_{k}}\right) \times \prod_{j=1}^{T} \varphi\left((-1)^{j+1} z\right) / \prod_{j=1}^{k-1} \varphi\left((-1)^{m_{j}+1} z\right),
\end{aligned}
$$

where $\varphi(z)=E\left[\mathrm{e}^{\mathrm{i} z \xi_{1}} 1_{\left\{\xi_{1}>0\right\}}\right]$ is the characteristic function of $\xi_{1}$ conditioned on $\left\{\xi_{1}>0\right\}$. From the relationships $\varphi(-z)=\overline{\varphi(z)} \quad$ and $\quad|\varphi(z)|^{2}=\varphi(z) \overline{\varphi(z)} \quad$ we have

$$
\begin{aligned}
& f_{t}(z)=(1-p)^{t} \\
& +\sum_{k=1}^{t} \sum_{m=k}^{t}|\varphi(z)|^{2\left[\frac{m-1}{2}\right]} \varphi(z)^{\left((-1)^{m}+1\right) / 2} p^{k}(1-p)^{t-m} \\
& \times \sum_{1 \leq m_{1}<m_{2}<\cdots<m_{k-1}<m} \prod_{j=1}^{k-1} \varphi\left((-1)^{m_{j}} z\right) /|\varphi(z)|^{2 k-2} .
\end{aligned}
$$

Notice that if only $r$ numbers of $m_{1}, m_{2}, \cdots, m_{k-1}$ are even then

$$
\prod_{j=1}^{k-1} \varphi\left((-1)^{m_{j}} z\right)=\varphi(z)^{r} \varphi(-z)^{k-1-r} .
$$

Therefore

$$
\begin{gathered}
\sum_{1 \leq m_{1}<m_{2}<\cdots<m_{k-1}<m} \prod_{j=1}^{k-1} \varphi\left((-1)^{m_{j}} z\right) \\
=\sum_{r=0}^{k-1} \varphi(z)^{r} \varphi(-z)^{k-1-r} P(m-1, k-1, r),
\end{gathered}
$$

where $P(m, k, r)$ is a number of possibilities to choose $r$ even and $k-r$ odd numbers from the set $1,2, \cdots, m$. Here $m \geq k \geq r$. There are only $[m / 2]$ even and $m-[m / 2]$ odd numbers among $1,2, \cdots, m$. Hence $P(m, k, r)=0$ if $r>[m / 2]$ or $k-r>m-[m / 2]$ and $P(m, k, r)=\left(\begin{array}{c}{[m / 2]} \\ r\end{array}\right)\left(\begin{array}{c}m-[m / 2] \\ k-r\end{array}\right)$ if $r \leq[m / 2]$ and

$k-r \leq m-[m / 2]$. Putting this expression into the Formula (3.7) yields

$$
\begin{aligned}
& f_{t}(z)=(1-p)^{t} \\
& +\sum_{k=1 m=k}^{t} \sum^{t} \frac{p^{k}(1-p)^{t-m}}{|\varphi(z)|^{2 k-2}}|\varphi(z)|^{2\left[\frac{m-1}{2}\right]} \varphi(z)^{\left((-1)^{m}+1\right) / 2} \\
& \times \sum_{r=\max \{0, k-m+[(m-1) / 2]\}}^{\min \{[(m-1) / 2], k-1\}}\left(\begin{array}{c}
{[(m-1) / 2]} \\
r
\end{array}\right)\left(\begin{array}{c}
m-1-[(m-1) / 2] \\
k-r
\end{array}\right) \\
& \cdot \varphi(z)^{r} \varphi(-z)^{k-1-r} .
\end{aligned}
$$

Using equation (3.6) one can compute joint characteristic function $f_{1}\left(z_{1}, z_{2}\right)$ of the moment $\sigma_{1}$ of the first trade and the $\operatorname{logarithm} \log \left(S^{(1)} / B_{0}\right)$ provided there was at least one trade, $\sigma_{1}<\infty$ in the following way

$$
\begin{aligned}
& f_{1}\left(z_{1}, z_{2}\right)=E\left[\exp \left(\mathrm{i} z_{1} \sigma_{1}+\mathrm{i} z_{2} \log \left(S^{(1)} / B_{0}\right)\right) 1_{\left\{\sigma_{1}<\infty\right\}}\right] \\
& =\sum_{k=1}^{T} \mathrm{e}^{\mathrm{i} k z_{1}} E\left[\mathrm{e}^{\mathrm{i} z_{2} \log \left(\sqrt{X_{k}} / B_{0}\right)} 1_{\left\{\sigma_{1}=k\right\}}\right] .
\end{aligned}
$$

Since

$\left\{\sigma_{1}=k\right\}=\left\{\xi_{1}>0\right\} \cap\left\{\xi_{2}>0\right\} \cap \cdots \cap\left\{\xi_{k-1}>0\right\} \cap\left\{\xi_{k}=0\right\}$ and the random variables $\xi_{1}, \xi_{2}, \cdots, \xi_{k}$ are independent then

$$
\begin{aligned}
& f_{1}\left(z_{1}, z_{2}\right) \\
= & \sum_{k=1}^{T} \mathrm{e}^{\mathrm{i} k z_{1}} E\left[\exp \left(\mathrm{i} z_{2} \sum_{j=1}^{k}(-1)^{j+1} \xi_{j}+\xi_{k} / 2\right) \prod_{j=1}^{k-1} 1_{\left\{\xi_{j}>0\right\}} 1_{\left\{\xi_{k}=0\right\}}\right] \\
= & \sum_{k=1}^{T} \mathrm{e}^{\mathrm{i} k z_{1}} \prod_{j=1}^{k-1} E\left[\mathrm{e}^{\mathrm{i} z_{2}(-1)^{j+1} \xi_{j}} 1_{\left\{\xi_{j}>0\right\}}\right] E\left[1_{\left\{\xi_{k}=0\right\}}\right] \\
= & p \sum_{k=1}^{T} \mathrm{e}^{\mathrm{i} k z_{1}} \prod_{j=1}^{k-1} \varphi\left((-1)^{j+1} z_{2}\right),
\end{aligned}
$$

where $\varphi(z)=E\left[\mathrm{e}^{\mathrm{i} z \xi_{1}} 1_{\left\{\xi_{1}>0\right\}}\right]$ is defined above. The relationships $\quad \varphi(-z)=\overline{\varphi(z)}$ and $|\varphi(z)|^{2}=\varphi(z) \overline{\varphi(z)}$ imply

$$
\begin{aligned}
& f_{1}\left(z_{1}, z_{2}\right) \\
& =p\left(\mathrm{e}^{\mathrm{i} z_{1}}+\mathrm{e}^{2 \mathrm{i}_{1}} \varphi\left(z_{2}\right)+\mathrm{e}^{3 \mathrm{i} z_{1}}\left|\varphi\left(z_{2}\right)\right|^{2}+\cdots\right. \\
& \left.+\mathrm{e}^{\mathrm{i} T z_{1}} \varphi\left(z_{2}\right)^{\left(1+(-1)^{T}\right) / 2}\left|\varphi\left(z_{2}\right)\right|^{2\left[\frac{T-1}{2}\right]}\right) \\
& =p\left(\mathrm{e}^{\mathrm{i} z_{1}} \sum_{j=0}^{\left[\frac{T-1}{2}\right]}\left(\mathrm{e}^{2 \mathrm{i} \mathrm{z}_{1}}\left|\varphi\left(z_{2}\right)\right|^{2}\right)^{j}\right. \\
& \left.+\mathrm{e}^{2 \mathrm{i} z_{1}} \varphi\left(z_{2}\right)^{\left[\frac{T}{2}\right]-1} \sum_{j=0}\left(\mathrm{e}^{2 \mathrm{i} z_{1}}\left|\varphi\left(z_{2}\right)\right|^{2}\right)^{j}\right) \\
& =\frac{p \mathrm{e}^{\mathrm{i} z_{1}}}{1-\mathrm{e}^{2 \mathrm{i} z_{1}}\left|\varphi\left(z_{2}\right)\right|^{2}}\left[1-\left(\mathrm{e}^{2 \mathrm{i} \mathrm{z}_{1}}\left|\varphi\left(z_{2}\right)\right|^{2}\right)^{\left[\frac{T-1}{2}\right]+1}\right. \\
& \left.\left.+\mathrm{e}^{\mathrm{i} z_{1}} \varphi\left(z_{2}\right)\left(1-\left(\mathrm{e}^{2 \mathrm{i}_{1}}\left|\varphi\left(z_{2}\right)\right|^{2}\right)^{\left[\frac{T}{2}\right]}\right]\right)\right]
\end{aligned}
$$


Similarly we can find joint characteristic function $f_{k}\left(z_{1}, z_{2}\right)$ of the difference $\sigma_{k}-\sigma_{k-1}$ between moments of $k$-th and $(k-1)$-st trades, $k=2,3, \cdots$ and the logarithm $\log \left(S^{(k)} / S^{(k-1)}\right)$ of the ratio between these trades provided there were at least $k$ trades, , $\sigma_{k}<\infty$.

$$
\begin{aligned}
& f_{k}\left(z_{1}, z_{2}\right) \\
= & E\left[\exp \left(\mathrm{i} z_{1}\left(\sigma_{k}-\sigma_{k-1}\right)+\mathrm{i} z_{2} \log \left(S^{(k)} / S^{(k-1)}\right)\right) 1_{\left\{\sigma_{k}<\infty\right\}}\right] \\
= & \sum_{j=k l=k-1}^{T} \sum_{l-1}^{j-1}\left[\mathrm{e}^{\mathrm{i} z_{1}(j-l)} \exp \left(\mathrm{i} z_{2} \sum_{r=l+1}^{j-1}(-1)^{r+1} \xi_{r}\right) 1_{\left\{\sigma_{k}=j\right\}} 1_{\left\{\sigma_{k-1}=l\right\}}\right] .
\end{aligned}
$$

Since $1_{\left\{\sigma_{k}=j\right\}} 1_{\left\{\sigma_{k-1}=l\right\}}=1_{\left\{\xi_{j}=0\right\}} \prod_{r=l+1}^{j-1} 1_{\left\{\xi_{r}>0\right\}} 1_{\left\{\sigma_{k-1}=l\right\}}$ and all multipliers here are independent then

$$
\begin{aligned}
& f_{k}\left(z_{1}, z_{2}\right) \\
= & \sum_{j=k l=k-1}^{T} \sum^{j-1} \mathrm{e}^{\mathrm{i} z_{1}(j-l)} \operatorname{Pr}\left[\sigma_{k-1}=l\right] \\
& \cdot E\left[\exp \left(\mathrm{i} z_{2} \sum_{r=l+1}^{j-1}(-1)^{r+1} \xi_{r}\right) 1_{\left\{\xi_{j}=0\right\}} \prod_{r=l+1}^{j-1} 1_{\left\{\xi_{r}>0\right\}}\right] \\
= & \sum_{j=k}^{T} \sum_{l=k-1}^{j-1} \mathrm{e}^{\mathrm{i} z_{1}(j-l)}\left(\begin{array}{c}
l-1 \\
k-2
\end{array}\right) p^{k}(1-p)^{l-k+1} \prod_{r=l+1}^{j-1} \varphi\left((-1)^{r+1} z_{2}\right),
\end{aligned}
$$

where $\varphi(z)=E\left[\mathrm{e}^{\mathrm{i} z \xi_{1}} 1_{\left\{\xi_{1}>0\right\}}\right]$ as above. After the changing the order of summation and summation indexes we have

$$
\begin{aligned}
& f_{k}\left(z_{1}, z_{2}\right) \\
& =\sum_{l=k-1}^{T-1}\left(\begin{array}{c}
l-1 \\
k-2
\end{array}\right) p^{k}(1-p)^{l-k+1} \sum_{j=1}^{T-l} \mathrm{e}^{\mathrm{i} z_{2} j} \prod_{r=1}^{j-1} \varphi\left((-1)^{r+l+1} z_{2}\right) .
\end{aligned}
$$

The same arguments as after Equality (3.8) lead to the following expression

$$
\begin{aligned}
& f_{k}\left(z_{1}, z_{2}\right) \\
& =\sum_{l=k-1}^{T-1} \frac{\left(\begin{array}{c}
l-1 \\
k-2
\end{array}\right) p^{k}(1-p)^{l-k+1} \mathrm{e}^{\mathrm{i} z_{1}}}{1-\mathrm{e}^{2 \mathrm{i} z_{1}}\left|\varphi\left(z_{2}\right)\right|^{2}}\left[1-\left(\mathrm{e}^{2 \mathrm{i} z_{1}}\left|\varphi\left(z_{2}\right)\right|^{2}\right)^{\left[\frac{T-l-1}{2}\right]+1}\right. \\
& \left.+\mathrm{e}^{\mathrm{i}_{1}} \varphi\left((-1)^{l} z_{2}\right)\left(1-\left(\mathrm{e}^{2 \mathrm{i} z_{1}}\left|\varphi\left(z_{2}\right)\right|^{2}\right)^{\left[\frac{T-l}{2}\right]}\right]\right]
\end{aligned}
$$

Now we consider one more simplest case.

Recall the expressions for $X_{t}, Y_{t}$ and $\tau_{t}$.

$$
\begin{gathered}
X_{t}=X_{0} \exp \left(\sum_{k=0}^{t-1}\left(\mu_{k}-v_{k}\right)\right), \\
\tau_{t}=\sup \left\{0<s \leq t: Y_{s}=1\right\}=\sup \left\{0<s \leq t: \xi_{t}=0\right\},
\end{gathered}
$$

where $Y_{t}=e^{\xi_{t}}, \xi_{t}=v_{t-1}$ for $t=2 k$ and $\xi_{t}=\mu_{t-1}$ if $t=2 k-1, k=1,2, \cdots$.

Assume that $\mu_{k}-v_{k}$ is a sequence of independent random variables. Then the power of exponent in the expression for $X_{t}$ is a random walk and $X_{t}$ is a discrete analogue of geometrical Brownian motion, which is classical choice for modeling of the price process. But in our model the price process describes by $X_{\tau}$, geometrical random walk computed at random time and the distributions of $X_{t}$ and $X_{\tau_{t}}$ can be completely different. We show that indeed this is the case and the distribution of $X_{\tau_{t}}$ is trivial.

Denote $\zeta_{t}=\mu_{t}-v_{t}$ : then we have

$$
X_{t}=X_{0} \exp \left(\sum_{k=0}^{t-1} \zeta_{k}\right) .
$$

Since $v_{2 k-1}=v_{2 k}$ and $\mu_{2 k}=\mu_{2 k+1}$ then $\zeta_{2 k}=\mu_{2 k}-v_{2 k-1}$ and $\zeta_{2 k-1}=\mu_{2 k-2}-v_{2 k-1}$. Therefore $\mu_{2 k}-v_{0}=\sum_{j=0}^{2 k}(-1)^{j} \zeta_{j}$ and $v_{2 k-1}-v_{0}=\sum_{j=0}^{2 k-1}(-1)^{j} \zeta_{j}$ which implies the following equality:

$$
Y_{t}=Y_{0} \exp \left(\sum_{k=0}^{t-1}(-1)^{k} \zeta_{k}\right) .
$$

From the meaning of process $Y_{t}$ we have $Y_{t} \geq 1$ for all $t \geq 0$ hence $\zeta_{t}$ for any $t \geq 0$ a.s. satisfy the following system of inequalities

$$
\sum_{k=0}^{t-1}(-1)^{k} \zeta_{k}+v_{0} \geq 0
$$

Denote the left side of the last inequality by $\kappa_{t}=\sum_{k=0}^{t-1}(-1)^{k} \zeta_{k}+v_{0}$. Then $\kappa_{t+1}=\kappa_{t}+(-1)^{t} \zeta_{t}$ and $\kappa_{t} \geq 0$ for all $t \geq 0$. It is evident that the random variables $\kappa_{t}$ and $\zeta_{t}$ are independent and $Y_{t}=1$ if and only if $\kappa_{t}=0$.

The following technical lemma will be needed.

Lemma 3.1. Let $\gamma$ and $\theta$ be two independent random variables. Then

$$
(\gamma+\theta)=\operatorname{ess} \inf (\gamma)+\operatorname{ess} \inf (\theta) .
$$

Proof. Recall the formula for distribution function of the sum of two independent random variables $\gamma$ and $\theta$

$$
F_{\gamma+\theta}(z)=\int_{-\infty}^{+\infty} F_{\gamma}(z-x) \mathrm{d} F_{\theta}(x),
$$

where $F_{\theta}(z)=\operatorname{Pr}[\theta \leq z]$ is the distribution function of the random variable $\theta$. Since $F_{\theta}(z)=0$ for all $z<\operatorname{ess} \inf (\theta)$ then

$$
F_{\gamma+\theta}(z)=\int_{\operatorname{ess} \inf (\theta)}^{+\infty} F_{\gamma}(z-x) \mathrm{d} F_{\theta}(x)=0,
$$

for all $z<\operatorname{ess} \inf (\gamma)+\operatorname{ess} \inf (\theta)$. This implies that ess inf $(\gamma+\theta) \geq \operatorname{ess} \inf (\gamma)+\operatorname{ess} \inf (\theta)$. Since the opposite inequality is obvious then we have the statement of the lemma. 
It follows from the non-negativity of $\kappa_{t}$ and lemma above that for all $t \geq 0$

$$
\text { ess } \inf \left(\kappa_{t}\right)=\sum_{k=0}^{t-1} \operatorname{essinf}\left((-1)^{k} \zeta_{k}\right)+v_{0} \geq 0 .
$$

The trade occurs at time $t$ if and only if $\kappa_{t}=0$, i.e. when the last inequality becomes in fact equality. In this case we have that $\zeta_{k}=(-1)^{k} \operatorname{ess} \inf \left((-1)^{k} \zeta_{k}\right)$ for any $k=1, \cdots, t-1$. Therefore

$$
\begin{gathered}
\tau_{t}=\sup \left\{0 \leq k \leq t: \zeta_{k}=(-1)^{k} \operatorname{essinf}\left((-1)^{k} \zeta_{k}\right) ;\right. \\
\left.\sum_{k=0}^{t-1} \operatorname{essinf}\left((-1)^{k} \zeta_{k}\right)+v_{0}=0\right\}
\end{gathered}
$$

And the price of the last trade is deterministic and is equal to the following expression

$$
\begin{aligned}
& S_{t}=\sqrt{X_{\tau_{t}}} \\
& =\sqrt{X_{0}} \exp \left(\frac{1}{2} \sum_{k=0}^{t-1}(-1)^{k} \operatorname{ess} \inf \left((-1)^{k} \zeta_{k}\right)\right) .
\end{aligned}
$$

In particular, if $\left((-1)^{k} \zeta_{k}\right)=0$ for all $k=0,1, \cdots$ then $t^{*}=\inf \left\{0<s \leq T: \zeta_{s} \neq 0\right\}-1$ is a last possible moment of trade. There is a trade at each time $t \leq t^{*}$ with the same price $S_{t}=\sqrt{X_{0}}$ and there are no trades at all after the moment $t^{*}$.

\section{The Connection to Continuous Time Analogue of the Model}

In this section we give an example of the agents' behavior such that the geometrical Brownian motion can be regarded as the limit of the price process

$S_{t}=\sqrt{X_{\tau_{t}}}$ with discrete time $t$. For this purpose let $\eta_{n}$ be a sequence of random variables describing the state of the real world (noise sequence). Assume that at each time $t$ the agents make their decisions about how to change bid or ask prices according to the history of the noise sequence before the present time $t$. For instance $\mu_{t}=f\left(\eta_{t}, \eta_{t-1}, \cdots, \eta_{0}\right)$ and $v_{t}=g\left(\eta_{t}, \eta_{t-1}, \cdots, \eta_{0}\right)$. The simplest case, with agents taking into account only the present value of noise $\eta_{t}$ was considered above.

Now we consider the case when the agents are taking into account only the present $\eta_{t}$ and previous $\eta_{t-1}$ information, $\mu_{t}=f\left(\eta_{t}, \eta_{t-1}\right)$ and $v_{t}=g\left(\eta_{t}, \eta_{t-1}\right)$ for even and odd moments. Assume that $\eta_{n}$ is a sequence of independent identically distributed random variables and set $\mu_{2 k+1}=\mu_{2 k}=\eta_{2 k}^{+}+\eta_{2 k-1}^{+}=\xi_{2 k+1}$ and $v_{2 k}=v_{2 k-1}=\eta_{2 k-1}^{-}+\eta_{2 k-2}^{-}=\xi_{2 k}$, where $x^{+}=\max \{0, x\}$ and $x^{-}=-\min \{0, x\}$.

For such $\mu$ and $v$ we can compute the distribution of $\tau_{t}$. For simplicity assume that
$\operatorname{Pr}\left[\eta_{1}>0\right]=\operatorname{Pr}\left[\eta_{1}<0\right]=1 / 2$. If there are no trades then

$$
\begin{aligned}
& \operatorname{Pr}\left[\tau_{t}=0\right] \\
& =\operatorname{Pr}\left[\xi_{1}>0 ; \xi_{2}>0 ; \cdots ; \xi_{t-1}>0 ; \xi_{t}>0\right] \\
& =\operatorname{Pr}\left[\left(\eta_{-1}^{+}>0 \bigcup \eta_{0}^{+}>0\right) \cap\left(\eta_{0}^{-}>0 \bigcup \eta_{1}^{-}>0\right)\right. \\
& \left.\quad \cap\left(\eta_{1}^{+}>0 \bigcup \eta_{2}^{+}>0\right) \cap\left(\eta_{2}^{-}>0 \bigcup \eta_{3}^{-}>0\right) \cap \cdots\right] \\
& =\operatorname{Pr}\left[\left(\eta_{-1}>0 \bigcup \eta_{0}>0\right) \cap\left(\eta_{0}<0 \bigcup \eta_{1}<0\right)\right. \\
& \left.\quad \cap\left(\eta_{1}>0 \bigcup \eta_{2}>0\right) \cap\left(\eta_{2}<0 \bigcup \eta_{3}<0\right) \cap \cdots\right]
\end{aligned}
$$

The last event happens if and only if the following condition is satisfied: for all $k=0,1, \cdots,[(t-1) / 2]$ at least one of the numbers $\eta_{2 k-1}$ and $\eta_{2 k}$ is positive and for all $m=0,1, \cdots,[(t-2) / 2]$ at least one of the numbers $\eta_{2 m}$ and $\eta_{2 m+1}$ is negative. If $\eta_{i}$ and $\eta_{i+1}$ have the same sign then the sign of other $\eta_{j}, j \neq i, i+1$ satisfying the condition above is uniquely determined. The condition above is also satisfied if $\eta_{i}$ and $\eta_{i+1}$ have the different signs for all $i=-1,2, \cdots, t-1$. Hence the number of possible choices of signs of $\eta_{i}$ satisfying condition above is equal to $t+2$, where $t$ is a number of choices of $i$ such that $\eta_{i}$ and $\eta_{i+1}$ have the same sign and 2 is number of possibilities that $\eta_{i}$ and $\eta_{i+1}$ have the different signs for all $i=-1,2, \cdots, t-1$. Since for any choice of signs of $\eta_{i}$ the probability is equal to $1 / 2^{t+1}$ then we get

$$
\operatorname{Pr}\left[\tau_{t}=0\right]=(t+2) \frac{1}{2^{t+1}} .
$$

Notice that if $\xi_{k}=0$ then $\xi_{k+1}>0$ and $\xi_{k-1}>0$ a.s. Indeed, for even $k$ we have $\xi_{k}=\eta_{k-2}^{-}+\eta_{k-1}^{-}$and since $\operatorname{Pr}\left[\xi_{1}=0\right]=0$ then $\left\{\xi_{k}=0\right\}=\left\{\eta_{k-2} \geq 0\right\} \cap\left\{\eta_{k-1} \geq 0\right\} \subset\left\{\eta_{k-1}>0\right\} \subset\left\{\xi_{k+1}>0\right\}$ a.s. For odd $k$ the proof is the same. The fact that $\xi_{k-1}>0$ if $\xi_{k}=0$ can be shown in the same way. Hence for $s=0,1, \cdots, t-1$ we get

$$
\begin{aligned}
& \operatorname{Pr}\left[\tau_{t}=t-s\right] \\
& =\operatorname{Pr}\left[\xi_{t-s}=0 ; \xi_{t-s+1}>0 ; \xi_{t-s+2}>0 ; \cdots ; \xi_{t}>0\right] \\
& =\operatorname{Pr}\left[\xi_{t-s}=0\right] \operatorname{Pr}\left[\xi_{t-s+2}>0 ; \cdots ; \xi_{t}>0\right]=\frac{1}{4} \operatorname{Pr}\left[\tau_{s-1}=0\right] \\
& =(s+1) \frac{1}{2^{s+2}} .
\end{aligned}
$$

Now consider $X_{t}$. From Equalities (3.3) and (3.4) we have

$$
X_{t}=B_{0}^{2} \exp \left(2 \sum_{k=0}^{t-2} \eta_{k}+2 \eta_{-1}^{+}+\varphi_{t-1}+\psi_{t-2}\right),
$$

where $\phi_{t}=\eta_{t}^{+}$if $t=2 m$ and $\phi_{t}=-\eta_{t}^{-}$if $t=2 m+1$, and $\psi_{t}=\eta_{t}^{-}$if $t=2 m$ and $\psi_{t}=-\eta_{t}^{+}$if $t=2 m+1$. Notice that the representation (4.1) is also true in the case when the random variables $\eta_{t}$ are not necessary independent and identically distributed. Since $\xi_{\tau_{t}}=0$, 
then $\varphi_{\tau_{t}-1}=\psi_{\tau_{t}-2}=0$ and from the last equation we deduce that

$$
S_{t}=\sqrt{X_{\tau_{t}}}=B_{0} \exp \left(\sum_{k=0}^{\tau_{t}-2} \eta_{k}+\eta_{-1}^{+}\right) .
$$

Let us compute joint characteristic function $f_{t}\left(z_{1}, z_{2}\right)$ of the sum $\sum_{k=0}^{\tau_{t}-2} \eta_{k}=\log \left(S_{t} / B_{0}\right)-\eta_{-1}^{+}$and $\tau_{t}$.

$$
\begin{aligned}
f_{t}\left(z_{1}, z_{2}\right) & =E\left[\exp \left(\mathrm{i} z_{1} \sum_{k=0}^{\tau_{t}-2} \eta_{k}+\mathrm{i} z_{2} \tau_{t}\right)\right] \\
& =\sum_{j=0}^{t} \mathrm{e}^{\mathrm{i} j z_{2}} E\left[\exp \left(\mathrm{i} z_{1} \sum_{k=0}^{j-2} \eta_{k}\right) 1_{\left\{\tau_{t}=j\right\}}\right] .
\end{aligned}
$$

It has been shown above that

$1_{\left\{\tau_{t}=j\right\}}=1_{\left\{\xi_{j}=0\right\}} 1_{\left\{\xi_{j+2}>0\right\}} 1_{\left\{\xi_{j+3}>0\right\}} \cdots 1_{\left\{\xi_{t}>0\right\}}$. Since $\xi_{k}$ depends on $\eta_{k-1}$ and $\eta_{k-2}$ only then

$$
\begin{aligned}
& f_{t}\left(z_{1}, z_{2}\right) \\
= & \sum_{j=0}^{t} \mathrm{e}^{\mathrm{i} z_{2}} E\left[\exp \left(\mathrm{i} z_{1} \sum_{k=0}^{j-2} \eta_{k}\right) 1_{\left\{\xi_{j}=0\right\}}\right] \\
& \cdot E\left[1_{\left\{\xi_{j+2}>0\right\}} 1_{\left\{\xi_{j+3}>0\right\}} \cdots 1_{\left\{\xi_{t}>0\right\}}\right] \\
= & \operatorname{Pr}\left[\tau_{t}=0\right]+\frac{\mathrm{e}^{\mathrm{i} z_{2}}}{4} \operatorname{Pr}\left[\tau_{t-2}=0\right] \\
+ & \sum_{j=2}^{t} \mathrm{e}^{\mathrm{i} z_{2}}\left(E\left[\mathrm{e}^{\mathrm{i} z_{1} \eta_{0}}\right]\right)^{j-2} E\left[\mathrm{e}^{\mathrm{i} z_{1} \eta_{j-2}} 1_{\left\{\xi_{j}=0\right\}}\right] \operatorname{Pr}\left[\tau_{t-j-1}=0\right] \\
= & (t+2) \frac{1}{2^{t+1}}+t \frac{\mathrm{e}^{\mathrm{i} z_{2}}}{2^{t+1}} \\
& +\sum_{j=2}^{t} \mathrm{e}^{\mathrm{i} j z_{2}}(t-j+1) \frac{1}{2^{t-j}} \varphi_{0}\left(z_{1}\right)^{j-2} E\left[\mathrm{e}^{\mathrm{i}_{1} \eta_{j-2}} 1_{\left\{\xi_{j}=0\right\}}\right],
\end{aligned}
$$

where $\varphi_{0}\left(z_{1}\right)=E\left[\mathrm{e}^{\mathrm{i} z_{1} \eta_{0}}\right]$ is the characteristic function of $\eta_{0}$.

The expression $E\left[\mathrm{e}^{\mathrm{i} z \eta_{j-2}} 1_{\left\{\xi_{j}=0\right\}}\right]$ can be simplified as follows. If $j=2 m$ then $\left\{\xi_{j}=0\right\}=\left\{\eta_{j-1}^{-}=0\right\} \cap\left\{\eta_{j-2}^{-}=0\right\}=\left\{\eta_{j-1} \geq 0\right\} \cap\left\{\eta_{j-2} \geq 0\right\}$ and

$$
E\left[\mathrm{e}^{\mathrm{i} z_{1} \eta_{j-2}} 1_{\left\{\xi_{j}=0\right\}}\right]=\frac{1}{2} E\left[\mathrm{e}^{\mathrm{i} z_{1} \eta_{j-2}} 1_{\left\{\eta_{j-2} \geq 0\right\}}\right] .
$$

For $j=2 m-1$ we have $\left\{\xi_{j}=0\right\}=\left\{\eta_{j-1} \leq 0\right\} \cap\left\{\eta_{j-2} \leq 0\right\}$. Therefore

$$
E\left[\mathrm{e}^{\mathrm{i} z_{1} \eta_{j-2}} 1_{\left\{\xi_{j}=0\right\}}\right]=\frac{1}{2} E\left[\mathrm{e}^{\mathrm{i}_{1} \eta_{j-2}} 1_{\left\{\eta_{j-2} \leq 0\right\}}\right] .
$$

Then the Equality (4.2) has the following form

$$
\begin{aligned}
& f_{t}\left(z_{1}, z_{2}\right) \\
& =(t+2) \frac{1}{2^{t+1}}+t \frac{\mathrm{e}^{\mathrm{i} z_{2}}}{2^{t+1}} \\
& +\sum_{j=2}^{t}(t-j+1) \frac{\mathrm{e}^{\mathrm{j} z z_{2}} \varphi_{0}\left(z_{1}\right)^{j-2}}{2^{t-j+1}} E\left[\mathrm{e}^{\mathrm{i} z_{1} \eta_{0}} 1_{\left\{(-1)^{j} \eta_{0} \geq 0\right\}}\right] \\
& =\frac{t+2}{2^{t+1}}+t \frac{\mathrm{e}^{\mathrm{i} z_{2}}}{2^{t+1}} \\
& +E\left[\mathrm{e}^{\mathrm{i} z_{1} \eta_{0}} 1_{\left\{\eta_{0} \geq 0\right\}}\right] \sum_{j=1}^{[t / 2]}(t-2 j+1) \frac{\mathrm{e}^{\mathrm{i} 2 j z_{2}} \varphi_{0}\left(z_{1}\right)^{2 j-2}}{2^{t-2 j+1}} \\
& +E\left[\mathrm{e}^{\mathrm{i} z_{1} \eta_{0}} 1_{\left\{\eta_{0} \leq 0\right\}}\right]^{[(t-1) / 2]} \sum_{j=1}(t-2 j) \frac{\mathrm{e}^{\mathrm{i}(2 j+1) z_{2}} \varphi_{0}\left(z_{1}\right)^{2 j-1}}{2^{t-2 j}} .
\end{aligned}
$$

Suppose at first that $t=2 m$. Then from the last equality we get

$$
\begin{aligned}
f_{t}\left(z_{1}, z_{2}\right)= & \frac{t+2}{2^{t+1}}+t \frac{\mathrm{e}^{\mathrm{i} z_{2}}}{2^{t+1}}+E\left[\mathrm{e}^{\mathrm{i} z_{1} \eta_{0}} 1_{\left\{\eta_{0} \geq 0\right\}}\right] \sum_{j=2}^{t}(t-j+1) \frac{\mathrm{e}^{\mathrm{i} z z_{2}} \varphi_{0}\left(z_{1}\right)^{j-2}}{2^{t-j+1}}-E\left[\operatorname{sgn}\left(\eta_{0}\right) \mathrm{e}^{\mathrm{i} z_{1} \eta_{0}}\right] \sum_{j=1}^{m-1} 2(m-j) \frac{\mathrm{e}^{\mathrm{i}(2 j+1) z_{2}} \varphi_{0}\left(z_{1}\right)^{2 j-1}}{2^{t-2 j}} \\
= & \frac{t+2}{2^{t+1}}+t \frac{\mathrm{e}^{\mathrm{i} z_{2}}}{2^{t+1}}+\frac{1}{2} \mathrm{e}^{\mathrm{i} \mathrm{i} z_{2}} \varphi_{0}\left(z_{1}\right)^{t-2} E\left[\mathrm{e}^{\mathrm{i} \eta_{1} \eta_{0}} 1_{\left\{\eta_{0} \geq 0\right\}}\right] \sum_{j=1}^{t-1} j\left(\frac{\mathrm{e}^{-\mathrm{i} z_{2}}}{2 \varphi_{0}\left(z_{1}\right)}\right)^{j-1} \\
& -\frac{1}{2} \mathrm{e}^{\mathrm{i}(t-1) z_{2}} \varphi_{0}\left(z_{1}\right)^{t-3} E\left[\operatorname{sgn}\left(\eta_{0}\right) \mathrm{e}^{\mathrm{i} z_{1} \eta_{0}}\right] \sum_{j=1}^{m-1} j\left(\frac{\mathrm{e}^{-2 \mathrm{i} z_{2}}}{4 \varphi_{0}\left(z_{1}\right)^{2}}\right)^{j-1} \\
= & \frac{t+2}{2^{t+1}}+t \frac{\mathrm{e}^{\mathrm{i} z_{2}}}{2^{t+1}}+E\left[\mathrm{e}^{\mathrm{i} z_{1} \eta_{0}} 1_{\left\{\eta_{0} \geq 0\right\}}\right]\left(\frac{2 \mathrm{e}^{\mathrm{i} z_{2}} \varphi_{0}\left(z_{1}\right)^{t}-1 / 2^{t-1}}{\left(2 \varphi_{0}\left(z_{1}\right)-\mathrm{e}^{-\mathrm{i} z_{2}}\right)^{2}}-\frac{t \mathrm{e}^{\mathrm{i} z_{2}}}{2^{t-1}\left(2 \varphi_{0}\left(z_{1}\right)-\mathrm{e}^{-\mathrm{i} z_{2}}\right)}\right) \\
& -E\left[\operatorname{sgn}\left(\eta_{0}\right) \mathrm{e}^{\mathrm{i} z_{1} \eta_{0}}\right]\left(\frac{8 \mathrm{e}^{\mathrm{i}(t-1) z_{2}} \varphi_{0}\left(z_{1}\right)^{t+1}}{\left(4 \varphi_{0}\left(z_{1}\right)^{2}-\mathrm{e}^{-2 \mathrm{i} z_{2}}\right)^{2}}-\frac{\mathrm{e}^{-\mathrm{i} z_{2}} \varphi_{0}\left(z_{1}\right)}{2^{t-3}\left(4 \varphi_{0}\left(z_{1}\right)^{2}-\mathrm{e}^{-2 \mathrm{i} z_{2}}\right)^{2}}-\frac{m \mathrm{e}^{\mathrm{i} z_{2}} \varphi_{0}\left(z_{1}\right)}{2^{t-3}\left(4 \varphi_{0}\left(z_{1}\right)^{2}-\mathrm{e}^{-2 \mathrm{i} z_{2}}\right)}\right) .
\end{aligned}
$$


Similarly we have for $t=2 m+1$

$$
\begin{aligned}
f_{t}\left(z_{1}, z_{2}\right)= & \frac{t+2}{2^{t+1}}+t \frac{\mathrm{e}^{\mathrm{i} z_{2}}}{2^{t+1}}+E\left[\mathrm{e}^{\mathrm{i} z_{1} \eta_{0}} 1_{\left\{\eta_{0} \leq 0\right\}}\right]\left(\frac{2 \mathrm{e}^{\mathrm{i} z z_{2}} \varphi_{0}\left(z_{1}\right)^{t}-1 / 2^{t-1}}{\left(2 \varphi_{0}\left(z_{1}\right)-\mathrm{e}^{-\mathrm{i} z_{2}}\right)^{2}}-\frac{t \mathrm{e}^{\mathrm{i} z_{2}}}{2^{t-1} 2 \varphi_{0}\left(z_{1}\right)-\mathrm{e}^{-\mathrm{i} z_{2}}}\right) \\
& +E\left[\operatorname{sgn}\left(\eta_{0}\right) \mathrm{e}^{\mathrm{i} z_{1} \eta_{0}}\right]\left(\frac{8 \mathrm{e}^{\mathrm{i}(t-1) z_{2}} \varphi_{0}\left(z_{1}\right)^{t+1}}{\left(4 \varphi_{0}\left(z_{1}\right)^{2}-\mathrm{e}^{-2 \mathrm{iz} z_{2}}\right)^{2}}-\frac{\mathrm{e}^{-2 \mathrm{i} z_{2}}}{2^{t-1}\left(4 \varphi_{0}\left(z_{1}\right)^{2}-\mathrm{e}^{-2 \mathrm{iz} z_{2}}\right)^{2}}-\frac{(m+1)}{2^{t-2}\left(4 \varphi_{0}\left(z_{1}\right)^{2}-\mathrm{e}^{-2 \mathrm{i} z_{2}}\right)}\right) .
\end{aligned}
$$

The last Equalities (4.3) and (4.4) allow one to obtain the characteristic function of a continuous time model analogous the process $S_{t}$ as the limit of the discrete time model.

For instance, consider the partition $0<h<2 h<\cdots<n h=1$ of the interval [0;1]. Let $t$ take values $0,1,2, \cdots, n$. Assume that $h \rightarrow 0$ and $t h \rightarrow s$, where $s \in[0 ; 1]$. If the noise sequence $\eta_{t}$ is Gaussian, $\varphi_{0}\left(z_{1}\right)=\mathrm{e}^{-h z_{1}^{2} / 2}$, then

$$
\lim _{h \rightarrow \infty} E\left[\mathrm{e}^{\mathrm{i} z_{1} \eta_{0}} 1_{\left\{\eta_{0} \leq 0\right\}}\right]=\lim _{h \rightarrow \infty} E\left[\mathrm{e}^{\mathrm{i} z_{1} \eta_{0}} 1_{\left\{\eta_{0} \geq 0\right\}}\right]=\frac{1}{2} .
$$

Hence from (4.3) and (4.4) we have

$$
F_{s}\left(z_{1}, z_{2}\right)=\lim _{\substack{h \rightarrow 0 \\ t h \rightarrow s}} f_{t h}\left(z_{1}, z_{2}\right)=\mathrm{e}^{-s z_{1}^{2} / 2} \mathrm{e}^{\mathrm{i} s z_{2}} .
$$

Therefore for Gaussian noise the continuous version of price process $S_{t}$ is a geometrical Brownian motion and $\tau_{t}=t$.

\section{Conclusions}

With this work we have set forth the structure for computing a price process from first principles of agent behavior in providing bid and ask quotes to a market. As well, we have provided some content by analyzing a basic case, that of a binomial assumption on the i.i.d. sequence $\left\{\xi_{t}\right\}$ recording the moments of trades. This assumption led to the specification of a geometric random walk computed in random time, and to the joint characteristic function $f_{k}\left(z_{1}, z_{2}\right)$ of the difference $\sigma_{k}-\sigma_{k-1}$ between moments of $k$-th and $(k-1)$-st trades, $k=1,2, \cdots$ and the logarithm $\log \left(S^{(k)} / S^{(k-1)}\right)$ of the ratio between these trades. The study culminated with an explicit expression for $S_{t}$, and implications for a parallel model in continuous time.

Next on the agenda is to explore alternative hypotheses on agent behaviors, and to perform simulations and other numerical work as necessary to establish a theory of consequential price processes.

\section{REFERENCES}

[1] A. Madhavan, “Market Microstructure: A Survey,” Jour- nal of Financial Markets, Vol. 3, No. 3, 2000, pp. 205258. doi:10.1016/S1386-4181(00)00007-0

[2] H. R. Stoll, Elsevier/North-Holland, Amsterdam, 2003.

[3] O. E. Barndorff-Nielsen, "Processes of Normal Inverse Gaussian Type,” Finance and Stochastics, Vol. 2, No. 1, 1998, pp. 41-68. doi:10.1007/s007800050032

[4] E. Eberlein and U. Keller, "Hyperbolic Distributions in Finance,” Bernoulli, Vol. 1, No. 3, 1995, pp. 281-299. doi:10.2307/3318481

[5] T. Bollerslev, "Financial Econometrics: Past Developments and Future Challenges," Journal of Econometrics, Vol. 100, No. 1, 2001, pp. 41-51. doi:10.1016/S0304-4076(00)00052-X

[6] R. F. Engle, “The Econometrics of Ultra-High-Frequency Data,” Econometrica, Vol. 68, No. 1, 2000, pp. 1-22. doi:10.1111/1468-0262.00091

[7] R. F. Engle and J. R. Russell, "Autoregressive Conditional Duration: A New Model for Irregularly Spaced Transaction Data," Econometrica, Vol. 66, No. 5, 1998, pp. 1127-1162. doi:10.2307/2999632

[8] J. Hasbrouck, "The Dynamics of Discrete Bid and Ask Quotes,” The Journal of Finance, Vol. 54, No. 6, 1999, pp. 2109-2142. doi:10.1111/0022-1082.00183

[9] O. Bondarenko, "Competing Market Makers, Liquidity Provision, and Bid-Ask Spreads,” Journal of Financial Markets, Vol. 4, 2001, pp. 269-308. doi:10.1016/S1386-4181(01)00014-3

[10] M. Schaden, “Quantum Finance,” Physica A, Vol. 316, No. 1-4, 2002, pp. 511-538. doi:10.1016/S0378-4371(02)01200-1

[11] S. Hermannn and P. Imkeller, “The Exit Problem for Diffusions with Time Periodic Drift and Stochastic Resonance,” Prepublication No. 01, Institut de Mathématiques Élie Cartan, Université Nancy 1, Lorraine, 2003.

[12] P. C. Kettler, O. M. Pamen and F. Proske, "On Local Times: Application to Pricing Using Bid-Ask,” Preprint \#13, University of Oslo, Oslo, 2009. www.paulcarlislekettler.net/docs/Oliv.pdf

[13] G. Di Nunno, B. Øksendal and F. Proske, "Malliavin Calculus for Lévy Processes with Applications to Finance,” Universitext, 2nd Edition, Springer, Berlin, 2009.

[14] Á. Cartea and T. Meyer-Brandis, "How Does Duration between Trades of Underlying Securities Affect Option Prices?” 2007. http://ssrn.com/abstract=1032714 